\title{
Effect of solution conductivity and electrode shape on the deposition of carbon nanotubes from solution using
}

\section{dielectrophoresis}

\author{
A Kashefian Naieni, and A Nojeh \\ Department of Electrical and Computer Engineering, The University of British Columbia, \\ Vancouver BC, V6T 1Z4, Canada
}

Email: anojeh@ece.ubc.ca

\begin{abstract}
Dielectrophoresis (DEP) is a popular technique for fabricating carbon nanotube (CNT) devices. The electric current passing through the solution during DEP creates a temperature gradient, which results in electrothermal fluid flow because of the presence of the electric field. CNT solutions prepared with various methods can have different conductivities and the motion of the solution because of the electrothermal phenomenon can affect the DEP deposition differently in each case. We investigated the effect of this movement in solutions with various levels of conductivity through experiments as well as numerical modeling. Our results show that electrothermal motion in the solution can alter the deposition
\end{abstract}


pattern of the nanotubes drastically for high conductivity solutions, while DEP remains the dominant force when a low conductivity (surfactant-free) solution is used. The extent of effectiveness of each force is discussed in the various cases and the fluid movement model is investigated using two- and threedimensional finite element simulations.

PACS numbers: 81.07.-b, 61.48.De, 81.16.Rf.

\section{Introduction}

Carbon nanotube (CNT)-based devices have been the focus of many research works throughout the past two decades. Despite the considerable interest in the applications of these devices, the issue of lack of reliability of the available fabrication methods for CNT devices at predetermined positions with desirable characteristics is yet to be resolved.

One of the popular methods for the fabrication of nano devices is deposition on pre-patterned electrodes using dielectrophoresis (DEP) [1-3]. This method has been widely used in the deposition of CNTs [4-7]. Although the mobility and conductivity of CNT devices formed using DEP are typically not as high as the ones made using direct chemical vapor deposition (CVD), the former have advantages such as lowtemperature processing, better positioning and the possibility of using solutions containing particular types of CNTs in the fabrication[8,9].

Although DEP is very promising for fabricating CNT devices, repeatability is still a challenge. There are various parameters that can affect the results, such as the frequency, duration and amplitude of the applied voltage, the shape of the electrodes and the solution properties. Several studies have investigated the effect of each of these parameters to varying degrees [10-12]; however, the effect of solution properties in the resulting deposition patterns has not been fully explored.

Among the media typically used for making CNT solutions, water is a popular one. The aqueous solutions commonly used either contain surfactants for suspending CNTs or use some form of pretreatment to functionalize the originally hydrophobic CNTs so they can dissolve in water $[13,14]$. Although both methods result in stable CNT solutions, these solutions have different physical and 
electrical properties. Even for the solutions made using surfactants, the percentage of surfactant dissolved in the solution can have a significant impact on the conductivity of the final solution.

The current passing through the solution during DEP creates a spatial temperature gradient, which leads to significant movement in parts of the solution.

Ramos et al. investigated the electrokinetics of the solution during DEP [15]. They discussed the effects of forces such as Brownian, buoyancy and electrothermal on the manipulation of sub-micrometer particles. Green et al. used experimental [16] and simulation [17] approaches to show the effect of the electrothermal force, caused by Joule heating and also light-induced heating, on the movement of the solution. In another work, Castellanos et al. investigated the main force affecting the suspended particles during DEP for a range of settings for the applied voltage. They concluded that AC electro-osmosis is dominant at low frequencies (up to a few $\mathrm{kHz}$ ), while electrothermal flow plays the primary role in the fluid movement at higher frequencies and voltages, and DEP governs the movement of the submicrometer particles for small systems at high frequencies [18].

A few works investigated the effects of solution properties, the movement in some parts of the CNT solution during DEP, and the effect of this movement on the deposition of CNTs. Lin et al. used numerical methods to show that electrothermal flow can considerably affect the attraction of the semiconducting CNTs toward the electrodes during DEP [19]. Burg et al. adopted a two-dimensional (2D) numerical electrokinetic framework and demonstrated that the temperature gradient resulting from Joule heating due to the current passing through the solution can have a significant effect on the fluid flow because of $\mathrm{AC}$ electroosmosis at lower frequencies and the electrothermal force at higher frequencies $[20]$.

Although the effect of the electrothermal force on creating movement in the solution has been shown previously, no systematic experimental report on the effect of solution conductivity on the deposition of CNTs with DEP is available. In the present work, we used CNT solutions with different conductivities, but similar in every other respect, to perform such a systematic study, with particular emphasis on how the deposition patterns are affected. We analyze the differences and explain them using finite element 
simulations. The effect of electrothermal movement in the solution caused by Joule heating was simulated for the various solutions. The simulation results show good agreement with the experiments and can shed light on the drastic changes that the solution conductivity can cause in the deposition patterns.

\section{Methodology}

In order to allow for a meaningful comparison between the results of DEP experiments using solutions with different conductivities, one needs solutions with the same number of suspended nanotubes per unit volume but with different concentrations of a surfactant material such as sodium dodecylbenzene sulfonate (SDBS). Normally, surfactant concentration affects the nanotube concentration [13]. To overcome this issue, we used a surfactant-free CNT solution, commercially available from NanoLab Inc. [21], where the suspended single-walled nanotubes are carboxylated prior to suspension in water, and have a length in the range of $1-5 \mu \mathrm{m}$ and an average diameter of $1.5 \mathrm{~nm}$. The initial concentration of the solution was $1 \mathrm{~g} /$ lit. By adding de-ionized water and desired amounts of SDBS, we prepared three solutions with $0,0.5$, and 1 weight percent (wt\%) surfactant with a CNT concentration of $50 \mathrm{mg} / \mathrm{lit}$. The role of the surfactant here is merely to change the conductivity of the solution. Although the surfactant might interact with the CNTs in the 0.5 , and $1 \mathrm{wt} \%$ solutions, this would not affect the CNT concentration in the solution as the CNTs are already separated and suspended. The conductivities of these solutions were 20,1010 , and $2100 \mu \mathrm{S} / \mathrm{cm}$, respectively, as measured by a YSI 3200 conductivity meter.

As mentioned before, the electrothermal phenomenon can lead to solution movement, which can adversely interfere with the movement of CNTs caused by the DEP force. As the conductivity of the solution increases, this interference is expected to be more significant. A series of experiments at various voltages were performed to investigate how these two forces shape the deposition of CNTs in DEP experiments using solutions with different conductivities and electrodes with different shapes. Three types of electrodes were used in the DEP experiments. The difference between the first two types of electrodes was their shape: in the first type, the electrodes were $4 \mu \mathrm{m}$ wide with a $4-\mu \mathrm{m}$ gap between the two opposite electrodes; the second type consisted of electrodes with a width of $50 \mu \mathrm{m}$ and a gap of $4 \mu \mathrm{m}$ 
(figures 1a and 1b). The third-type electrodes had a very sharp tip of $85 \mathrm{~nm}$ in width with $2 \mu \mathrm{m}$ of distance between the opposite electrodes (figure 1c). All the electrodes were connected to large pads that could be used to connect the device to external circuitry through micro-probes.

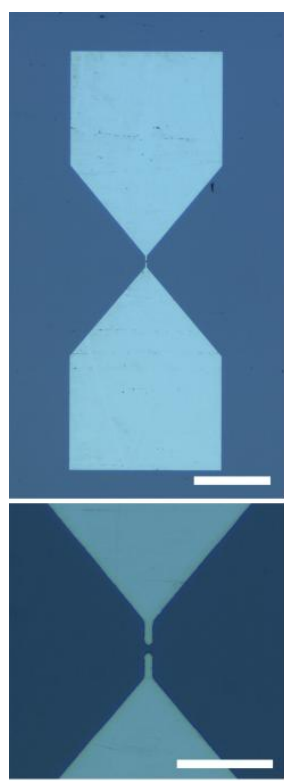

(a)

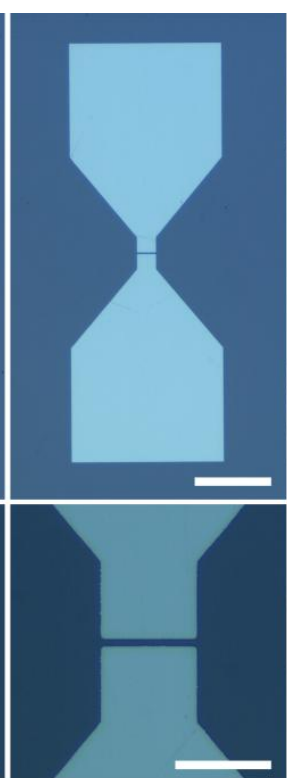

(b)

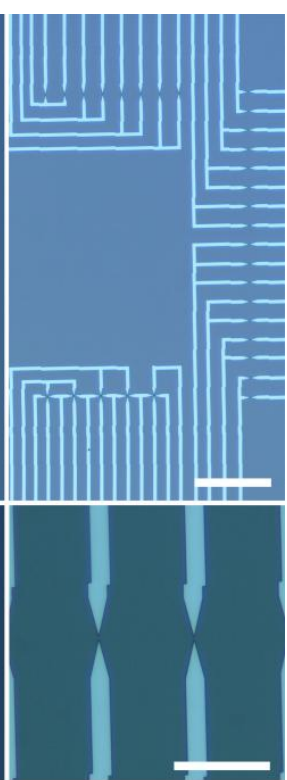

(c)

Figure 1. The electrodes used for the DEP experiments. The bottom row images show magnified views of the main device area (the electrode edges and the gap). (a) narrow, (b) wide, and (c) sharp electrodes. The scale bar is $200 \mu \mathrm{m}$ on the top-row and $50 \mu \mathrm{m}$ on the bottom-row images.

Fabrication of narrow and wide electrodes: the electrodes were made using normal photolithography, electron-beam metal deposition process and lift off, respectively, on a highly P-doped $<100>$ silicon wafer with $2 \mu \mathrm{m}$ of oxide on the top surface. (The deposited metal electrodes included $20 \mathrm{~nm}$ of chromium for adhesion and $50 \mathrm{~nm}$ of palladium.)

Fabrication of sharp electrodes: these electrodes were fabricated using the nano-SOI process available from CMC Microsystems. In this process, a silicon-on-insulator substrate is used. The process consists of electron-beam lithography, deposition of chromium and palladium layers with a total thickness of $40 \mathrm{~nm}$, and metal and silicon etch using chlorine-based reactive ion etching and photoresist stripping. 
DEP experiments: The DEP experiments were performed starting with pouring and spreading a $10-\mu$ lit drop of solution on a chip containing 24 pairs of electrodes for narrow and wide electrode designs. For sharp electrodes, the design has electrodes with various gap distances on the same chip. In the case of the solution with no surfactant, the solution could not be spread at the same level as the other two solutions

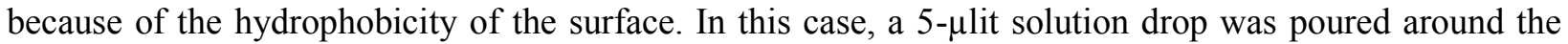
target electrodes. Electrodes with different shapes were used. A signal generator was connected to one of the electrodes and the other electrode was grounded through a $200 \mathrm{~K} \Omega$ resistor in parallel with an oscilloscope. The duration of each experiment was 1 minute. This gives enough time to the CNTs to be deposited on the electrodes and, at the same time, is not excessively long to the point of having nanotubes cover everywhere and mask the difference that using various voltages make. In all of the experiments, the frequency of the applied voltage was set to $5 \mathrm{MHz}$, which is a typical DEP frequency used in several previous reports $[4,10,22]$. After each experiment, the chip was rinsed with DI water and blow-dried using nitrogen gas. The samples were imaged using a Hitachi S4700 field-emission scanning electron microscope at $1 \mathrm{kV}$ of primary beam acceleration voltage.

\section{Results and discussion}

Figure 2 shows the results of the DEP experiments using narrow electrodes at voltages ranging from 3.5 to $5.5 \mathrm{~V}$. For the solution with no surfactant (figure 2a), the CNTs deposit in the entire area between the electrodes even at the lowest applied potential. At low voltages, the CNTs deposit in the central regions between the electrodes where the electric field has its maximum value. As the voltage is increased, they start covering the entire gap. The number of deposited CNTs increases as the applied voltage is increased. Therefore, there is a direct relation between the applied voltage and the deposition force on the CNTs. This shows that the main force here is DEP and that the electrothermal force, which is also a function of the applied potential, is almost negligible. 
The morphology of the deposited CNTs is drastically different for the cases where the solution contains surfactant. Instead of covering the whole gap, the CNTs are concentrated more on the edges of the electrodes. For the solution with $0.5 \mathrm{wt} \%$ surfactant (figure $2 \mathrm{~b}$ ), and specially at lower voltages, few nanotubes bridge the gap. However, at higher voltages and also for the 1 -wt $\%$-surfactant case (figure $2 \mathrm{c}$ ), the CNTs mostly cover the electrodes' surroundings. There is no direct relationship between the applied voltage - and, therefore, the magnitude of the DEP force - and the number of deposited nanotubes. The eletrothermal force is thus more dominant in these cases and is expected to be more pronounced for the more conductive solutions and at higher voltages. 

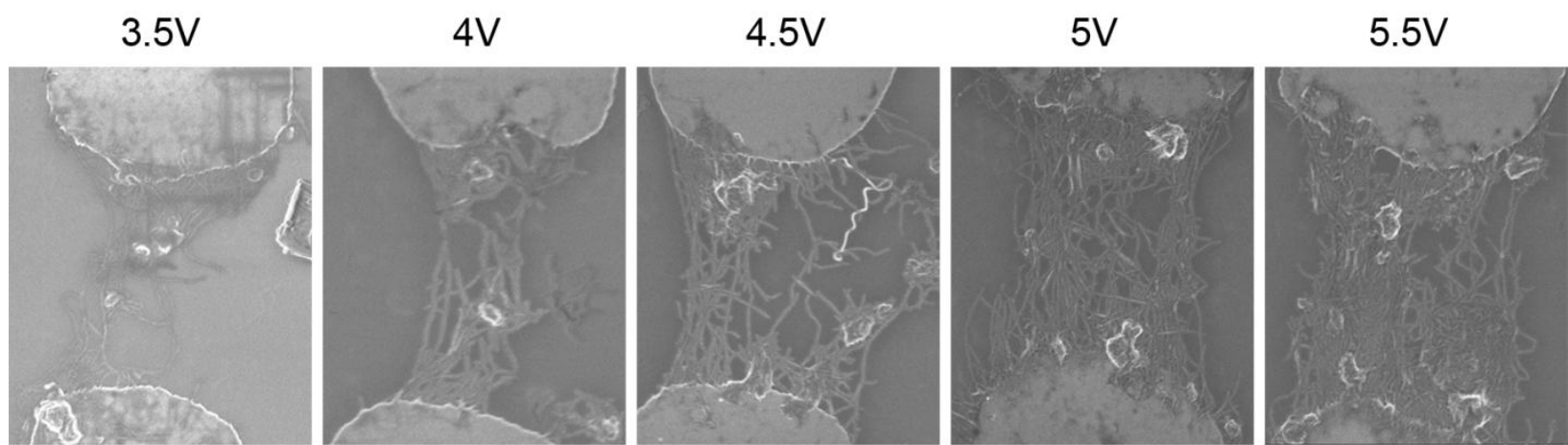

(a)
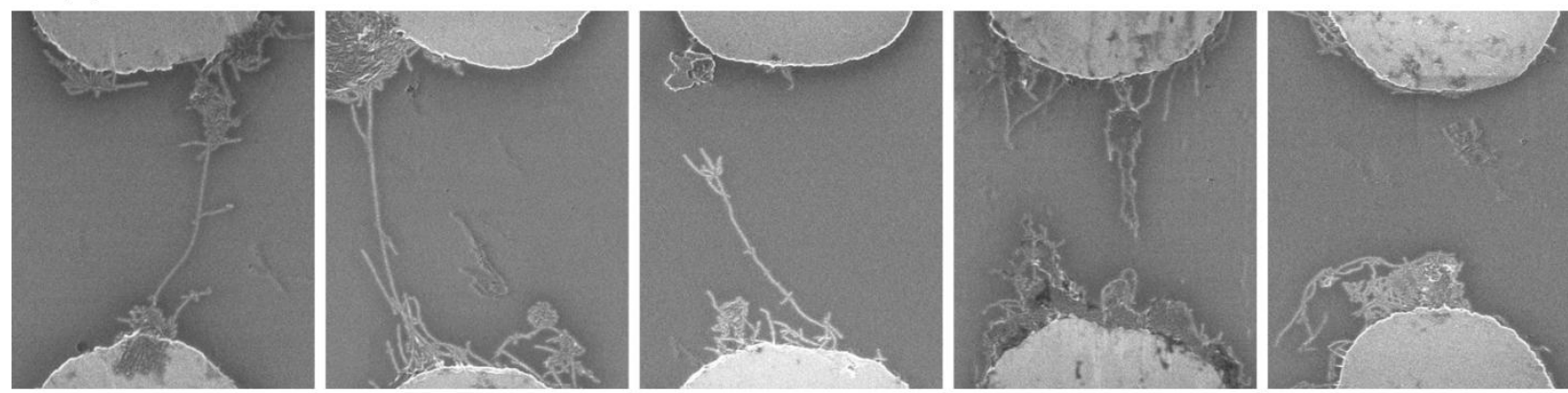

(b)
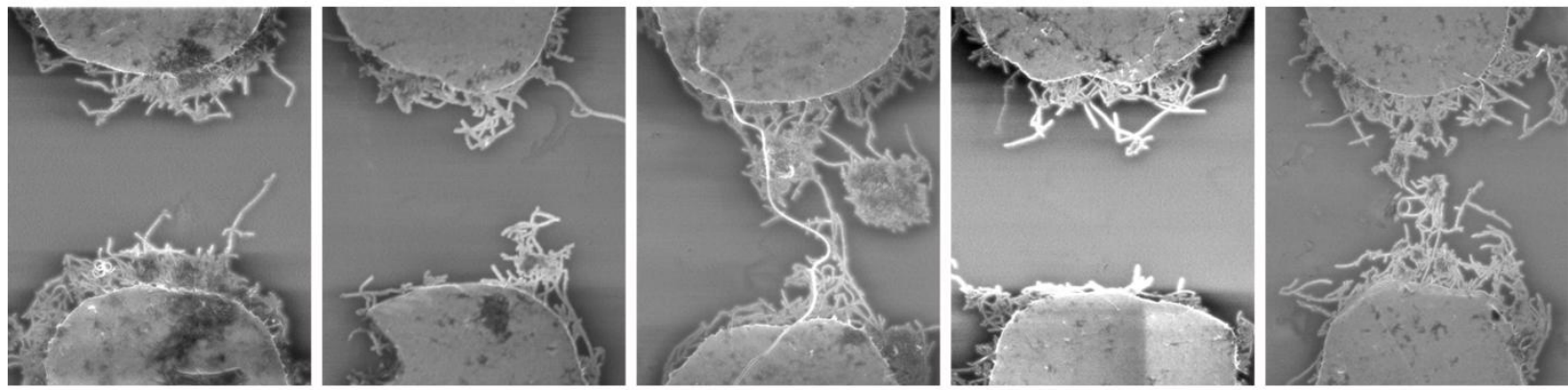

(c)

Figure 2. Scanning electron micrographs of the devices made from solutions with (a) 0 , (b) 0.5 , and (c) 1 $\mathrm{wt} \%$ surfactant, at voltages ranging from $3.5 \mathrm{~V}$ to $5.5 \mathrm{~V}$. The gap between the electrodes is $4 \mu \mathrm{m}$ long.

Figure 3 shows the results of DEP experiments using a $5.5-\mathrm{V}$ potential difference applied to wide electrodes. While the CNTs from the surfactant-free solution fill the gap between the electrodes completely, for the other two solutions the CNTs mostly cover the electrodes' edges. Occasionally, at places where the edges of the electrodes are already covered, some CNTs may deposit in the gap and bridge the CNTs on the two sides. 
The experiments on the wide and narrow electrodes were performed at least for three separate devices for each voltage setting and solution conductivity shown in figure 2 and figure 3, for a total of over 54 devices. The results of all the experiments showed deposition patterns similar to what is shown here. The only exceptions were 3 devices with narrow electrodes using the solution with no surfactant, where no CNT or few CNTs were deposited, most likely due to the lack of proper electrical contact between the delicate micro-probes and the electrodes.

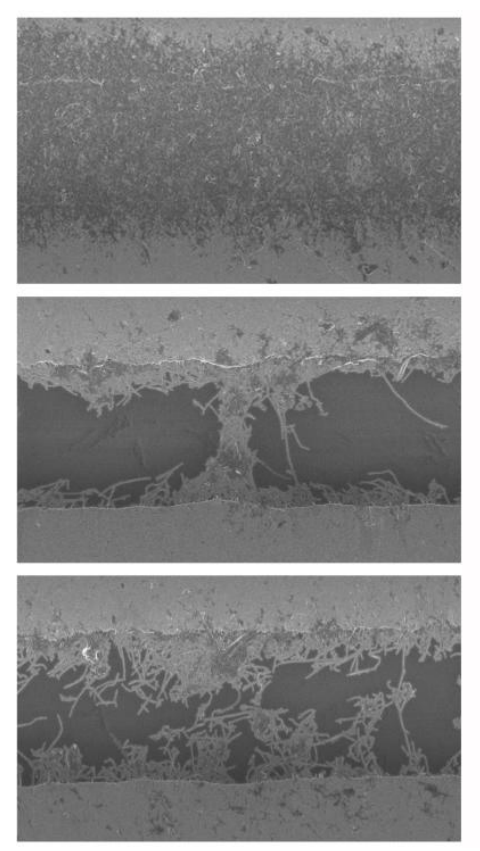

(a)

(b)

Figure 3. Scanning electron micrographs of the devices made with wide electrodes, using solutions with (a) 0 , (b) 0.5 , and (c) $1 \mathrm{wt} \%$ surfactant. The gap between the electrodes is $4 \mu \mathrm{m}$ long.

In the case of DEP experiments with wide electrodes, in the gap and away from the corners there is no change in the DEP and electrothermal forces in the direction parallel to the electrode edges. Therefore, the deposition is expected to be more uniform compared to that in the narrow electrodes, in which case the CNTs are more concentrated in the central region of the gap. The latter point is even more evident if sharp electrodes - for which the central region is practically a point - are used (figure 4): the gap is bridged by 
two CNTs when the solution has no surfactant and the DEP force plays the primary role, but there is no CNT deposited in the case of the solution with $1 \%$ surfactant.

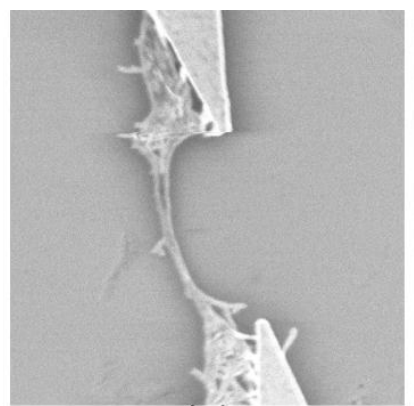

(a)

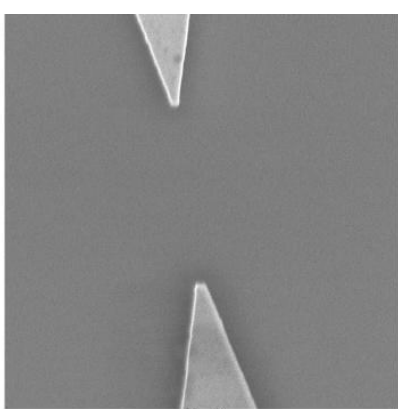

(b)

Figure 4. Scanning electron micrographs of the devices made using sharp electrodes with (a) surfactantfree, and (b) $1 \mathrm{wt} \%$ surfactant solutions. A $3 \mathrm{~V}$ potential difference was applied to the electrodes. The gap between the electrodes is $2 \mu \mathrm{m}$ long.

To understand the effectiveness of electrothermal flow in each case, a series of two- and threedimensional (2-D and 3-D) finite element simulations were performed using COMSOL Multiphysics [23] to find the direction and magnitude of the fluid flow in the solution. In the 3-D models, the shapes of the electrodes could be directly implemented in the model, making the simulations more realistic, and the solution movement could be investigated in all three directions.

The electric potential and field in the system were calculated using phasor-based quasi-static Maxwell's equations. The electrodes were set to appropriate potential values and the back gate was grounded. Electric insulation was applied to the boundaries of the system and, therefore, the perpendicular component of the current density was set to zero on all the outer boundaries.

When a potential is applied between the electrodes, the current passing through the solution as a result of the electric field can raise the temperature around the gap region between the electrodes because of Joule heating,

$$
Q=<\sigma|\vec{E}|^{2}>=\frac{1}{2} \sigma E^{2}
$$


, where $\mathrm{Q}$ is the generated power, $\sigma$ is the solution conductivity, $\overrightarrow{\mathrm{E}}$ is the electric field vector, and $\mathrm{E}$ is the AC electric field amplitude. This effect rapidly establishes a stationary temperature field with a negligible oscillating component [15]. The convective motion of the solution is negligible in microsystems and, therefore, the energy balance equation can be written as

$$
k \nabla^{2} T+Q=0
$$

, in which $\mathrm{k}$ is the thermal conductivity of the medium and $\mathrm{T}$ is the temperature $[15,20]$. The boundary conditions applied for the thermal calculations were different in 2-D and 3-D simulations. For the 2-D case, the system boundaries were far away from the heat source and their temperature was supposed to be very close to that of the surroundings; therefore, in this case the temperature of the boundaries was set equal to the ambient temperature. On the other hand, due to computational limitations, the boundaries of the 3-D models were closer to the heat source, and could affect the results unrealistically if they were set to a constant temperature. To avoid this problem, instead we set the normal temperature gradient component at the boundaries to zero. In both cases, the electrodes were assumed to be thick enough to conduct the heat easily and stay at room temperature.

The gradient in the temperature leads to gradients in conductivity and permittivity. These spatial changes in the presence of an electric field give rise to a body force on the fluid known as electrothermal force. The time-averaged electrothermal force per unit volume can be approximated using

$$
<\overrightarrow{f_{E}}>=-\frac{1}{2}\left[\left(\frac{\nabla \sigma}{\sigma}-\frac{\nabla \varepsilon}{\varepsilon}\right) \cdot \vec{E} \frac{\varepsilon \vec{E}}{1+(\omega \tau)^{2}}+\frac{1}{2}|\vec{E}|^{2} \nabla \varepsilon\right]
$$

, in which $\varepsilon$ is the permittivity of the solution and $\tau=\varepsilon / \sigma$ is the charge relaxation time in the solution [15]. This expression can be rewritten as a function of temperature gradient as

$$
<\overrightarrow{f_{E}}>=\frac{1}{2}\left[(\alpha-\beta)(\nabla T . \vec{E}) \frac{\varepsilon \vec{E}}{1+(\omega \tau)^{2}}-\frac{1}{2}|\vec{E}|^{2} \varepsilon \alpha \nabla T\right]
$$

, where $\alpha=\partial \varepsilon / \varepsilon \partial T=-0.4 \%$ and $\beta=\partial \sigma / \sigma \partial T=2 \%$ per Kelvin for an aqueous solution [24]. This formula was manually entered into COMSOL. Using the temperature profile calculated by COMSOL based on equation 2 as explained before, the electrothermal body force was computed for all the meshes 
covering the model.In order to calculate the velocity of the fluid at each point, the electrothermal force was used in the Navier-Stokes equation, combined with the mass conservation equation,

$$
\eta \nabla^{2} \vec{u}-\nabla p+\vec{f}=0
$$

and

$$
\nabla \cdot \vec{u}=0
$$

, in which $\eta$ is the dynamic viscosity of the solution, $\mathrm{p}$ is the pressure, $\vec{u}$ is the velocity vector and $\vec{f}$ is the general volumetric force (here, the electrothermal force, $\overrightarrow{f_{E}}$, calculated as described above).

The electrothermal force formula has two terms. The first term on the right hand side of (3), which represents the Coulomb force, is dominant at low frequencies. The dielectric force (the second term) is the major component at high frequencies. The transition frequency, at which the dominant term changes, is of the order of the inverse of the relaxation time. The two components are in different directions and, therefore, the direction of the electrothermal flow is different at low and high frequencies $[15,17,20]$.

The 2-D model consisted of a $200 \mu \mathrm{m}$ x $150 \mu \mathrm{m}$ segment representing the solution above the $70-\mathrm{nm}$ electrodes and $2-\mu \mathrm{m}$ oxide. The electrodes were set at $\pm \mathrm{V} / 2(\mathrm{~V}$ is the applied potential difference between the electrodes) and the back gate was grounded. The gap between the electrodes was $4 \mu \mathrm{m}$, as in the experiments.

Figure 5 shows the results of the 2-D simulations of the fluid movement at $5 \mathrm{~V}$. The diagrams showing temperature profiles for different cases are presented in the supplementary document (figure S1). The direction and magnitude of the flow are shown in this figure. Not only the velocities in the surfactant-free solution are orders of magnitude smaller than those in the solutions with surfactant, but also, and importantly, they are in the opposite direction in the two cases. The reason is the much higher relaxation time of the surfactant-free solution, which makes the transition frequency considerably lower than in the other two cases. The maximum velocity for the surfactant-free solution is on the order of a tenth of a micrometer per second, which means that considering the size of the system, the solution is almost stationary. 
For the solutions containing surfactant, electrothermal flow is at maximum velocity in the close vicinity of the gap, and it creates a circular motion in a large portion of the bulk of the solution. The direction of the flow is from above the gap toward the edges of the electrodes, and then it continues outward over the electrodes and eventually back over the gap. The long-range movement of the CNTs is governed by the fluid flow and the CNTs are pushed toward the edges of the electrodes. This can explain the difference between the experimental results of the various cases. While the solution is almost stationary in the case of the surfactant-free solution, in the presence of surfactants (leading to considerable conductivity), electrothermal flow carries the CNTs with very high velocities toward the edges of the electrodes and, after that, away from the gap, which gives a short time to the DEP force to make the CNTs deposit only close to the edge of the electrodes.

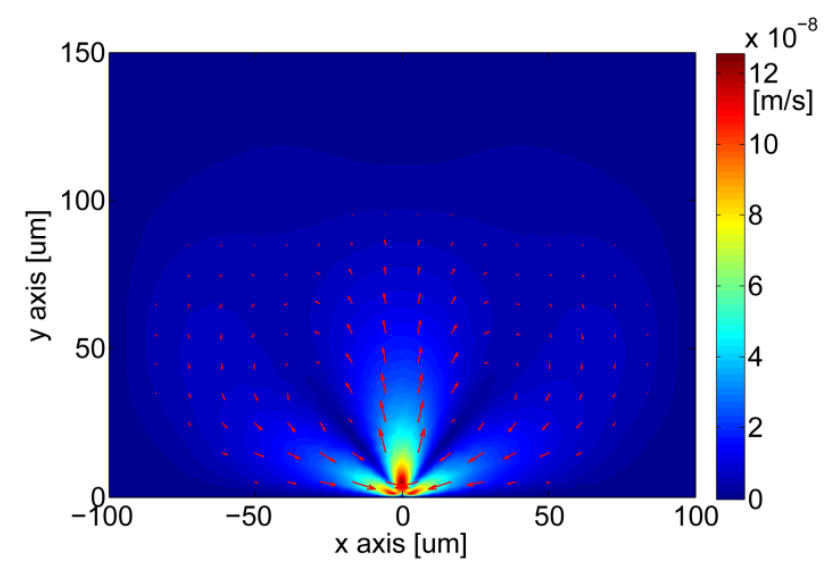

(a) 


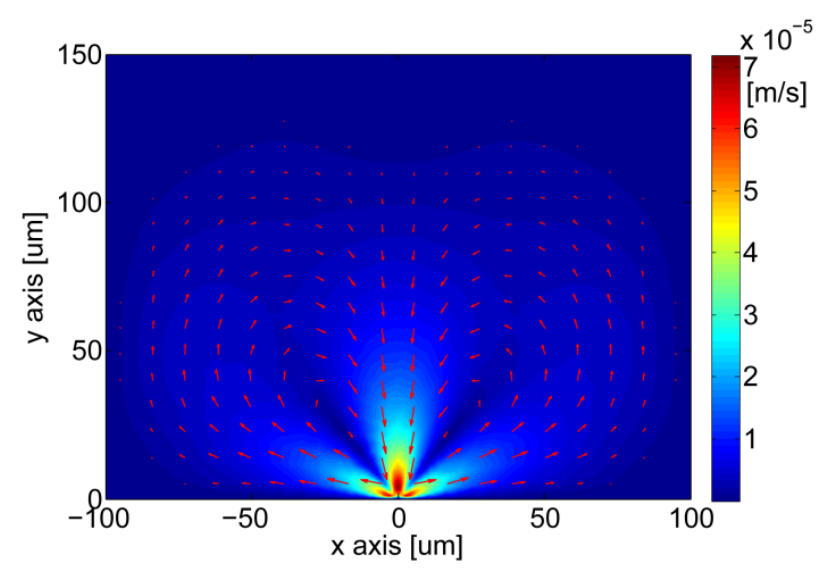

(b)

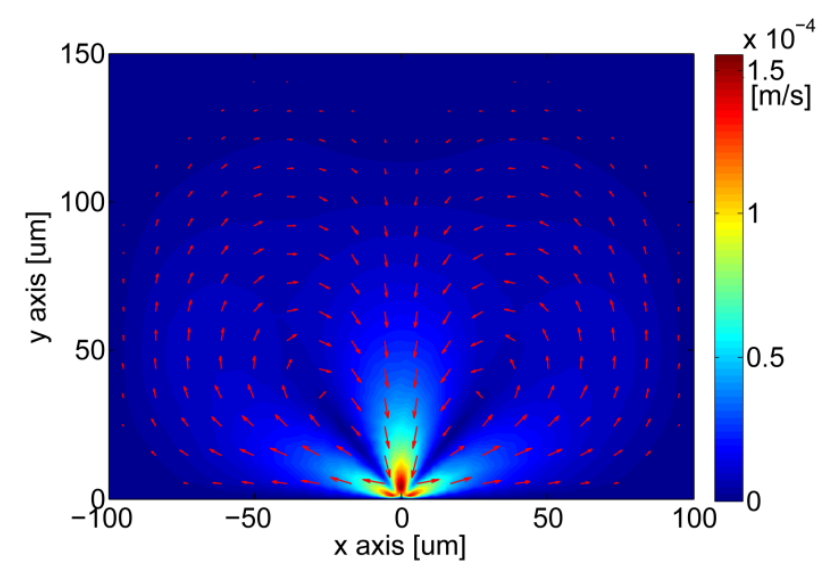

(c)

Figure 5. (color online) The fluid flow induced by the electrothermal force for (a) 0 , (b) 0.5 , and (c) 1 wt $\%$ surfactant solutions. The electrode edges are at -2 and $2 \mu \mathrm{m}$ on the horizontal axis. The colors show the magnitude of the velocity in the entire bulk of the solution. The vectors are logarithmically related with the solution velocity at each point.

In order to understand the changes in the fluid velocity as a function of the applied potential, a series of simulations were performed for both $0.5-\mathrm{wt} \%$ and $1-\mathrm{wt} \%$ solutions with the same range of applied voltages as in the experiments. Figure 6 shows the magnitude of the velocity of the electrothermal flow at a point $10 \mu \mathrm{m}$ above the center of the gap. The results show a non-linear increase in the velocity as the 
applied voltage increases. This can explain the presence of some CNTs in the middle of the gap at low applied voltages with the medium conductivity solution. At higher voltages or for the higher-conductivity solution, the velocity is considerably higher and the nanotubes are pushed toward the edges and away from the middle of the gap.

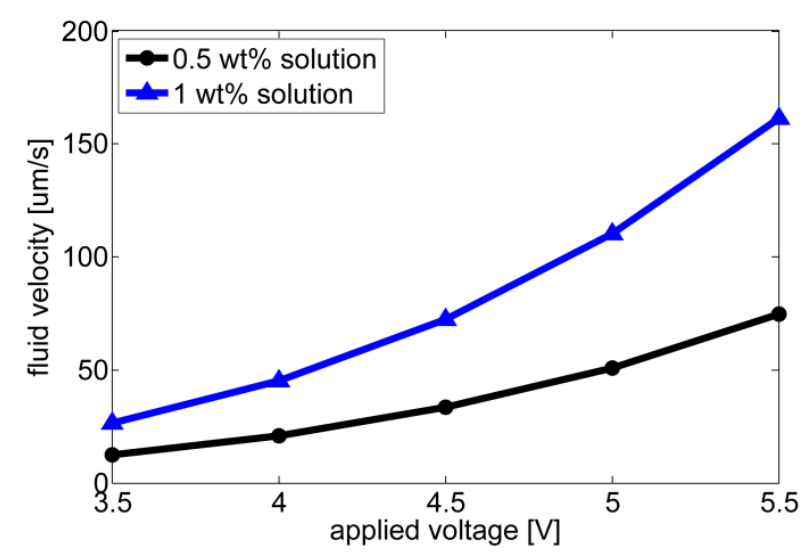

Figure 6. (color online) the magnitude of the fluid velocity as a function of the applied voltage. The black line with circles shows the velocity values for the solution with $0.5 \mathrm{wt} \%$ surfactant. The blue line with triangles shows the values for the solution containing $1 \mathrm{wt} \%$ surfactant.

The DEP force depends on the gradient of the square of the field and, for a particle with ellipsoidal shape, can be calculated using

$$
<\vec{f}_{D E P}>=\frac{\pi a b c}{3} \varepsilon_{m} \operatorname{Re}\left\{\frac{\varepsilon_{p}^{*}-\varepsilon_{m}^{*}}{\varepsilon_{m}^{*}}\right\} \nabla|\vec{E}|^{2}
$$

, in which $\mathrm{a}, \mathrm{b}$, and $\mathrm{c}$ are half of the lengths of the major ellipsoid axes and $\varepsilon_{p}^{*}$ and $\varepsilon_{m}^{*}$ are the particle (in our case CNT) and medium's permittivities, respectively. Having the DEP force field in the simulation medium, one can investigate the overall movement of the CNTs as a result of the electrothermal motion of the fluid and the DEP force using

$$
\vec{u}_{C N T}=\vec{u}+\frac{\vec{F}_{D E P}}{f} .
$$


, where $\vec{u}$ is the velocity vector of the fluid movement and $f$ is the friction factor. For a rod-shaped particle moving randomly in the solution, $f$ can be calculated using

$$
f=\frac{3 \pi \eta l}{\ln \left(\frac{l}{r}\right)}
$$

, with 1 and $\mathrm{r}$ being the length and radius of the rod [25].

Figure 7 shows the overall movement of the nanotubes in the medium for the case of 1 -wt $\%$-surfactant solution under a $5-\mathrm{V}$ applied potential. It can be seen that the long range movement of the CNTs is dictated by the electrothermal flow, but the DEP force is dominant near the gap and captures the CNTs as they pass in the close vicinity of the gap. The maximum of color-bar in figure 7 is set to $200 \mu \mathrm{m} / \mathrm{s}$ to allow for more details to be visible over the entire figure. It should be noted that the velocity of nanotubes as a result of the DEP force is much higher close to the edges of the gap, whereas the fluid velocity is zero on the boundaries because of the no-slip boundary condition. Without the DEP force, the nanotubes would move with the fluid because of the electrothermal force and, although they would approach the gap, they would not deposit there. Figures $7 \mathrm{~b}$ and 7c show the magnified version of the nanotubes' velocities near the gap as a result of the electrothermal flow (figure 7b) and electrothermal and DEP combined (figure $7 \mathrm{c}$ ) to ease the comparison. 


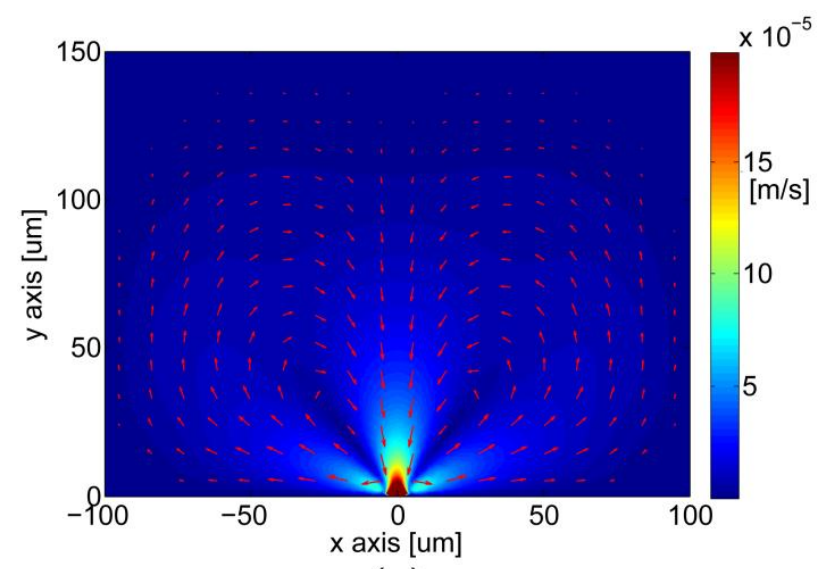

(a)

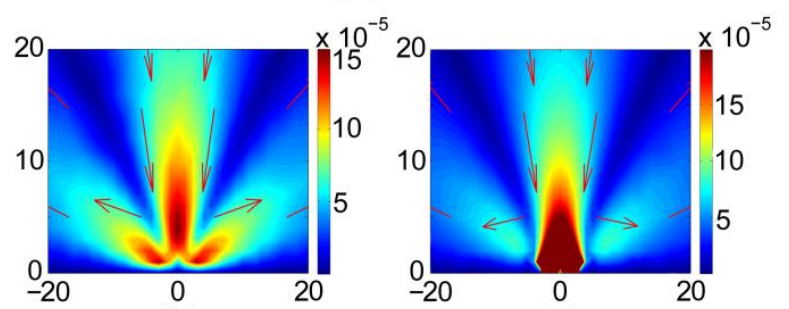

(b)

(c)

Figure 7. (color online) (a) the overall velocity of movement of the CNTs as a result of electrothermal flow and DEP force for the 1-wt\%-surfactant solution at $5 \mathrm{~V}$, (b) the movement of nanotubes in the 1$\mathrm{wt} \%$ solution in the presence of electrothermal flow only, near the gap region (magnified view of figure 5c near the gap), (c) the magnified view of figure $7 \mathrm{a}$ near the gap region. The vector lengths are logarithmically related with the solution velocity at each point.

The 2-D simulations cannot take the shape of the electrodes into account. To gain a better understanding of the fluid movement in the third direction, three types of electrodes -narrow, wide, and sharp- were simulated using 3-D models. The simulation structure consisted of a pair of electrodes over 2 $\mu \mathrm{m}$ of silicon dioxide, plus a $16 \times 20 \times 10 \mu \mathrm{m}$ medium representing the solution. Figure $\mathrm{S} 2$ (a), (b), and (c) in the supplementary document show the 3-D models.

Although these simulations capture the 3-D nature of the forces, their computationally expensive nature puts a limit on the size of the simulation box and, therefore, the boundaries have a stronger impact on the results compared to the larger-size 2-D simulations. The temperature gradient created by the wide 
electrodes is higher than in the case of the narrow electrodes and also sharp electrodes due to the larger total current passing through the solution. This higher temperature gradient causes faster movements in the solution. Figure S3 in the supplementary document shows the temperature profiles for different cases.

Figure 8 shows the fluid motion pattern in the plane of symmetry between the two electrodes (x-z plane in figure S2). The fluid has a very homogeneous movement straight from the top toward the gap when the width of the electrodes is considerably larger than the gap. In this case, the fluid has an in-plane movement in the planes parallel to the $y-z$ plane (figure 8a). For the narrow electrodes, the fluid not only moves in the $\mathrm{z}$ direction, but also moves in the $\mathrm{x}$ direction toward the gap (figure $8 \mathrm{~b}$ ). For the sharp electrodes, the movement is even more concentrated toward the line connecting the tips of the electrodes (figure8c).

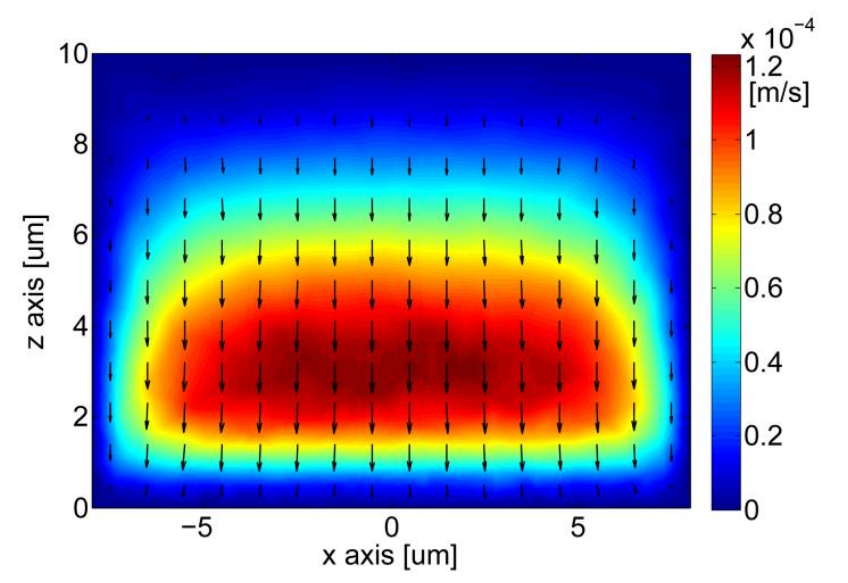

(a)

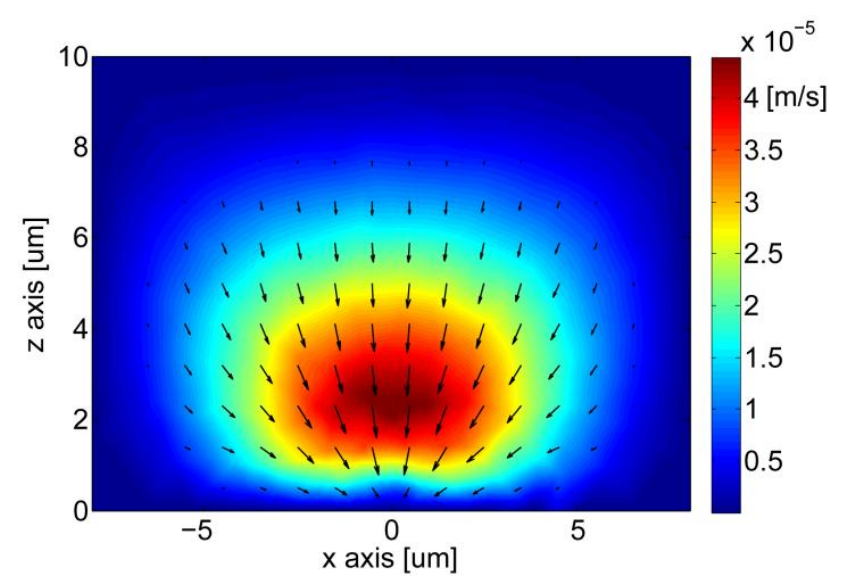


(b)

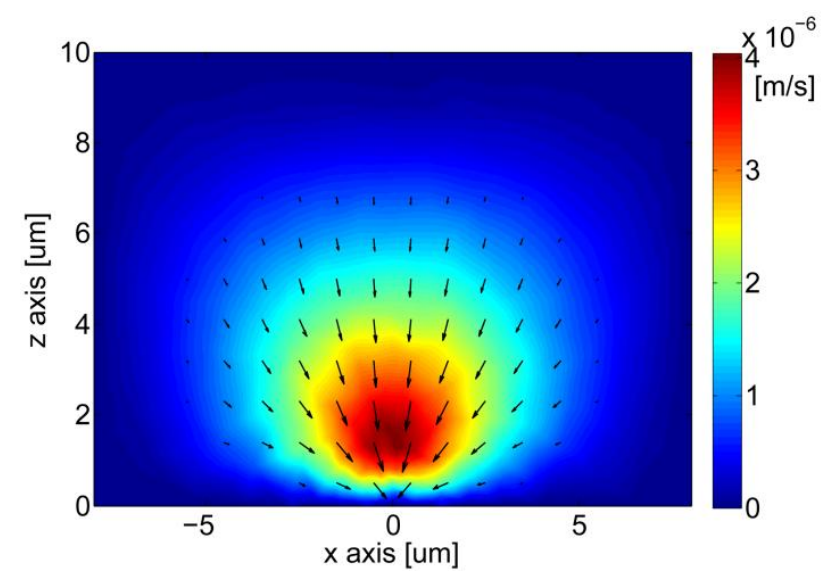

(c)

Figure 8. The fluid flow pattern in the $\mathrm{x}-\mathrm{z}$ plan in the middle of the gap of (a) wide, (b) narrow, and (c) sharp electrodes. The vector lengths are logarithmically related with the solution velocity at each point. Note that the $\mathrm{x}$ direction in this figure is different from the $\mathrm{x}$ direction in figures 5 and 7.

As it was discussed, the simulation results are consistent with the experimental observations, and demonstrate that the electrothermal force plays a key role in the deposition of nanotubes especially for solutions in which surfactant materials are used for the separation of the CNTs.

\section{Conclusion}

Dielectrophoresis experiments were performed and accompanied by simulations in order to study the effect of the electrothermal force on the deposition pattern of CNTs from solutions with various conductivities, using electrodes with different shapes. The results show that in the case of the surfactantfree solution, there is negligible movement in the solution under applied voltage. Experimentally, this results in the deposition of CNTs all around the gap between the electrodes. The presence of surfactants in the solution increases the conductivity and, therefore, the Joule heating effect. The resulting electrothermal force can be of primary significance in the morphology of the deposited nanotube 
collection on the surface; The CNTs deposit mostly on the electrode edges. Simulations show that the reason behind this is the movement of the fluid from the upper regions of the solution toward the gap, and then its deflection toward the edges of the electrodes and onto the electrodes' surfaces on each side.

The direction of the electrothermal movement of the solution depends on the shape of the electrodes. For electrodes with a width considerably larger than their gap, the flow takes place in planes perpendicular to the gap edge. For electrodes with narrow widths, the movement has a 3-D profile.

Taking the electrothermal force into account appears to be of primary importance in designing DEP deposition processes for CNTs.

\section{Acknowledgement}

We would like to acknowledge CMC Microsystems and, in particular, Imed Zine-El-Abidine, for the provision of products and services that facilitated this research, including the NanoSOI fabrication process. We thank the Natural Sciences and Engineering Research Council, the BCFRST Foundation/British Columbia Innovation Council, the Canada Foundation for Innovation and the British Columbia Knowledge Development Fund for financial support. 


\section{References}

1. Pething R 2010 Review Article- Dielectrophoresis: Status of the Theory, Technology, and Applications Biomicrofluidics 4022811

2. Khondaker S I, Luo K, and Yao Z 2010 The Fabrication of Single-Electron Transistors Using Dielectrophoretic Trapping of Individual Gold Nanoparticles Nanotechnology 21095204

3. Mohseni Kiasari N, and Servati P 2011 Dielectrophoresis-Assembled ZnO Nanowire Oxygen Sensors IEEE Electron Device Lett. 32 982-4

4. Banerjee S, White B E, Huang L, Rego B J, O’Brien S, and Herman I P 2006 Precise Positioning of Single-Walled Carbon Nanotubes by AC Dielectrophoresis J. Vac. Sci. Technol. B 24 3173-8

5. Vijayaraghavan A et al 2010 Toward Single-Chirality Carbon Nanotube Device Arrays ACS Nano 4 $2748-54$

6. Sarker B K, Shekhar S, and Khondaker S I 2011 Semiconducting Enriched Carbon Nanotube Aligned Arrays of Tunable Density and Their Electrical Transport Properties ACS Nano 5 6297-305.

7. Shekhar S, Stokes P, and Khondaker S I 2011 Ultrahigh Density Alignment of Carbon Nanotube Arrays by Dielectrophoresis ACS Nano 5 1739-46

8. Arnold M S, Green A A, Hulvat J F, Stupp S I, and Hersam M C 2006 Sorting Carbon Nanotubes by Electronic Structure Using Density Differentiation Nat. Nanotechnol. 1 60-5

9. Ghosh S, Bachilo S M, and Weisman R B 2010 Advanced Sorting of Single-Walled Carbon Nanotubes by Nonlinear Density-Gradient Ultracentrifugation Nat. Nanotechnol. 5 443-50

10. Arun A, Salet P, and Ionescu A M 2009 A Study of Deterministic Positioning of Carbon Nanotubes by Dielectrophoresis J. Electron. Mater. 38 742-9 
11. Shkhar S, Stokes P, and Khondaker S I 2011 Ultrahigh Density Alignment of Carbon Nanotube Arrays by Dielectrophoresis ACS Nano 5 1739-46

12. Duchamp M, Lee K, Dwir B, Seo J W, Kapon E, Forro L, and Magarez A 2010 Controlled Positioning of Carbon Nanotubes by Dielectrophoresis: Insights into the Solvent and Substrate Role $A C S$ Nano 4 279-84

13. Blanch A J, Leneha C E, and Quinton J S 2010 Optimizing Surfactant Concentrations for Dispersion of Single-Walled Carbon Nanotubes in Aqueous Solution J. Phys. Chem. B 114 9805-11

14. Kharisov B I, Kharissova O V, Gutierrez H L, and Mendez U O 2009 Recent Advances on the Soluble Carbon Nanotubes. Ind. Eng. Chem. Res. 48 572-90

15. Ramos A, Morgan H, Green N G, and Castellanos A 1998 AC Electrokinetics: A Review of Forces in Microelectrode Structure J. Phys. D: Appl. Phys. 31 2338-53

16. Green N G, Ramos A, Gonzalez A, Castellanos A, and Morgan H 2000 Electric Field Induced Fluid Flow on Microelectrodes: the Effect of Illumination J. Phys. D: Appl. Phys. 33 L13-7

17. Green N G, Ramos A, Gonzalez A, Castellanos A, and Morgan H 2001 Electrothermally Induced Fluid Flow on Microelectrodes J. Electrost. 53 71- 87

18. Castellanos A, Ramos A, Gonzalez A, Green N G, and Morgan H 2003 Electrohydrodynamics and Dielectrophoresis in Microsystems: Scaling Laws J. Phys. D: Appl. Phys. 36 2584-97

19. Lin Y, Shiomi J, Maruyama S, and Amberg G 2007 Electrothermal Flow in Dielectrophoresis of Single-Walled Carbon Nanotubes. Phys. Rev. B 76045419

20. Burg B R, Bianco V, Schneider J, and Poulikakos D 2010 Electrokinetic Framework of Dielectrophoretic Deposition Devices J. Appl. Phys. 107124308

21. http://www.nano-lab.com 
22. Seo H W, Han C S, Choi D G, Kim K S, and Lee Y H 2005 Controlled Assembly of Single SWNTs Bundle Using Dielectrophoresis Microelectron. Eng. 81 83-9

23. http://www.comsol.com

24. Lide D R 2009 CRC Handbook of Chemistry and physics, 92th ed. (CRC Press: London)

25. Morgan H, and Green N G 2003 AC Electrokinetics: Colloids and Nanoparticles (Research Studies Pree LTD.: Baldock) 